\title{
Monocytes accumulate in the airways of children with fatal asthma
}

\author{
Eguiluz-Gracia, Ibon
}

2018-12

Eguiluz-Gracia , I, Malmström , K, Dheyauldeen , S A , Lohi , J , Sajantila , A , Aalokken , R , Sundaram , A Y M , Gilfillan, G D , Makela , M , Baekkevold, E S \& Jahnsen , F L 2018 , ' Monocytes accumulate in the airways of children with fatal asthma ', Clinical and Experimental Allergy, vol. 48 , no. 12 , pp. 1631-1639 . https://doi.org/10.1111/cea.13265

http://hdl.handle.net/10138/307714

https://doi.org/10.1111/cea.13265

publishedVersion

Downloaded from Helda, University of Helsinki institutional repository.

This is an electronic reprint of the original article.

This reprint may differ from the original in pagination and typographic detail.

Please cite the original version. 


\title{
Monocytes accumulate in the airways of children with fatal asthma
}

\author{
Ibon Eguíluz-Gracia $^{1}$ | Kristiina Malmstrom ${ }^{2}$ | Sinan Ahmed Dheyauldeen ${ }^{3}$ | \\ Jouko Lohi ${ }^{4}$ | Antti Sajantila ${ }^{5}$ Ragnhild Aaløkken ${ }^{6}$ | Arvind Y. M. Sundaram ${ }^{6}$ | \\ Gregor D. Gilfillan $^{6}$ | Mika Makela ${ }^{2}$ | Espen S. Baekkevold ${ }^{1}$ (i) | Frode L. Jahnsen ${ }^{1}$
}

\footnotetext{
${ }^{1}$ Department of Pathology and Centre for Immune Regulation, Oslo University Hospital-Rikshospitalet and University of Oslo, Oslo, Norway

${ }^{2}$ Department of Allergy, Helsinki University Central Hospital, Helsinki, Finland

${ }^{3}$ Department of Otorhinolaryngology, Head and Neck Surgery, Oslo University HospitalRikshospitalet and University of Oslo, Oslo, Norway

${ }^{4}$ Department of Pathology, Helsinki University Central Hospital, Helsinki, Finland

${ }^{5}$ Department of Forensic Medicine, Hjelt Institute, University of Helsinki, Helsinki, Finland

${ }^{6}$ Department of Medical Genetics, Oslo University Hospital and University of Oslo, Oslo, Norway

Correspondence

Espen S. Baekkevold, Department of Pathology and Centre for Immune Regulation, Oslo University HospitalRikshospitalet and University of Oslo, Oslo, Norway.

Email: espbae@rr-research.no
}

\begin{abstract}
Background: Activated T helper type 2 (Th2) cells are believed to play a pivotal role in allergic airway inflammation, but which cells attract and activate Th2 cells locally have not been fully determined. Recently, it was shown in an experimental human model of allergic rhinitis (AR) that activated monocytes rapidly accumulate in the nasal mucosa after local allergen challenge, where they promote recruitment of Th2 cells and eosinophils.
\end{abstract}

Objective: To investigate whether monocytes are recruited to the lungs in paediatric asthma.

Methods: Tissue samples obtained from children and adolescents with fatal asthma attack ( $n=12)$, age-matched non-atopic controls $(n=9)$ and allergen-challenged AR patients $(n=8)$ were subjected to in situ immunostaining.

Results: Monocytes, identified as CD68+S100A8/A9+ cells, were significantly increased in the lower airway mucosa and in the alveoli of fatal asthma patients compared with control individuals. Interestingly, cellular aggregates containing CD68+S100A8/A9+ monocytes obstructing the lumen of bronchioles were found in asthmatics (8 out of 12) but not in controls. Analysing tissue specimens from challenged AR patients, we confirmed that co-staining with CD68 and S100A8/A9 was a valid method to identify recently recruited monocytes. We also showed that the vast majority of accumulating monocytes both in the lungs and in the nasal mucosa expressed matrix metalloproteinase 10 , suggesting that this protein may be involved in their migration within the tissue.

Conclusions and clinical relevance: Monocytes accumulated in the lungs of children and adolescents with fatal asthma attack. This finding strongly suggests that monocytes are directly involved in the immunopathology of asthma and that these proinflammatory cells are potential targets for therapy.

KEYWORDS

fatal asthma, MMP10, monocytes, paediatric asthma 


\section{1 | INTRODUCTION}

The prevalence of asthma in childhood is continuously increasing and imposes a significant burden to society in terms of medication, hospitalization days, school absenteeism and life-years lost. ${ }^{1,2}$ The inflammatory response of allergic asthma is characterized by aberrant activation of allergen-reactive T helper 2 (Th2) cells secreting IL-4, IL-5 and IL-13 and accumulation of activated eosinophils, both of which contribute to increased reactivity of the airways. T-cell responses in the airways are controlled by a network of antigen-presenting cells (APCS), which consist of monocytes, macrophages and dendritic cells (DCs). ${ }^{3}$ In animal models of allergic asthma, it has been shown that conventional (c)DCs residing in the tissue capture inhaled allergens and migrate to the draining lymph nodes where they activate rare allergen-specific $T$ cells. These $T$ cells subsequently home back to the site of allergen exposure to become reactivated by local APCs, including resident DCs. ${ }^{4}$ Compatible with this notion, studies of allergic asthma patients have shown that CD1c+ cDCs accumulate in the respiratory mucosa after bronchial allergen challenge with a concomitant decrease in $\mathrm{CD} 1 \mathrm{c}+\mathrm{cDCs}$ in peripheral blood, ${ }^{5,6}$ suggesting that increased recruitment of $\mathrm{CDCs}$ from the circulation occurs during allergic inflammation. However, studies in mice also showed that monocytes accumulating in the lungs played a pivotal role in the allergic inflammatory reaction both by secreting cytokines and chemokines ${ }^{7}$ and by reactivating allergen-specific effector $\mathrm{T}$ cells. ${ }^{8}$ In line with this, it was reported that in allergic children requiring hospitalization due to asthma exacerbations, activated monocytes increased in the circulation. ${ }^{9}$ However, whether monocytes are recruited to the airways in allergic asthmatics has not been investigated.

In an in vivo model of allergic rhinitis (AR), we recently found that circulating monocytes were rapidly (within hours) recruited to the nasal mucosa following local allergen challenge and remained the major APC subset in the nasal mucosa during 1 week with daily allergen exposure. ${ }^{10}$ Global transcriptome analysis of APCs (including monocytes) isolated from the challenged mucosa showed increased expression of genes for several Th2-associated chemokines concomitant with recruitment of $\mathrm{T}$ cells and eosinophils. ${ }^{10}$ This finding suggested that accumulating inflammatory monocytes may play an important role in $A R$, and led us to investigate whether recruitment of monocytes is a prominent feature also in asthma.

\section{2 | MATERIAL AND METHODS}

\section{1 | Asthmatic patients and non-atopic controls}

Post-mortem tissue samples were obtained from a death certificate study on fatal asthma in children and adolescents $(n=12)$ during 1976-1998 in Finland. ${ }^{11,12}$ Median age at death was 3.1 years (range 0.9-19.5). Of the individuals with reliable information, 4 of 6 cases had parents that were asthma sufferers. Nine of 12 asthmatic children displayed atopy. The median delay of anti-inflammatory medication from the beginning of asthma symptoms was 0.8 years, and only 5 of 12 patients were taking inhaled corticosteroids at the time of the fatal asthma attack (Table 1). The airways of the asthmatics showed typical signs of chronic Th2 inflammation, ${ }^{13}$ including a marked thickening of the basement membrane of the epithelium and plugs of mucus in the lumen of bronchi and bronchioles. ${ }^{12}$ Lung biopsies from 15 age-matched non-atopic individuals (median age 5.5 years, range 0.1-16.4) with accidental death between 2006 and 2010 were received from medico-legal autopsies and served as controls. The necropsy specimens were obtained $<48$ hours' post-mortem and were subsequently fixed in formalin and embedded in paraffin (FFPE) for in situ immunostaining. Approval for this study was obtained from the Finnish Ministry of Social Affairs and Health, National Supervisory Authority for Welfare and Health and Ethics Committee for Hospital for Children and Adolescents (220/13/03/03/ 2009).

\subsection{Allergic rhinitis patients}

Birch pollen AR patients ( $n=8,20-25$ year old, 5 women) were included. All patients fulfilled the Allergic Rhinitis and its Impact on Asthma (ARIA) criteria for persistent, moderate/severe $A R^{14}$ and had been symptomatic for at least the last three pollen seasons. All patients had a positive skin prick test to birch pollen. Five subjects were also sensitized to grass, mugwort, ragweed, mites and/or cat, dog or horse dander. Any other type of nasal disorder was excluded by examining the patients' medical histories and by clinical examination including nasal endoscopy. All patients were challenged according to the protocol shown in Figure S1, and the provocation was performed out of the pollen season. No patient experienced nasal allergic symptoms in the 3 months before the provocation or received any medication during the challenge period, and all of them were otherwise healthy. None of the participants were smokers. The study was approved by the Regional Committee for Medical and Health Research Ethics in Norway (2010/1753), and all patients gave their informed written consent.

\subsection{Nasal allergen challenge}

The nasal challenge procedure has been described in detail elsewhere, ${ }^{15}$ and the challenge protocol is shown in Figure S1. A solution of pollen extract $(30 \mathrm{HEP} / \mathrm{mL}$, Test de Provocación Betula alba, Laboratorios Leti, Spain) was delivered once a day for two consecutive days into one nostril with a hand-driven pump spray giving a defined volume of $50 \mu \mathrm{L}$. This model of in vivo experimentally induced human AR induces typical AR symptoms, and the recruitment of eosinophils, as demonstrated in previous studies from our group. $^{10,15}$

\subsection{Nasal symptoms}

The patients were instructed to score each single nasal symptom (sneezing, rhinorrhoea and nasal blockage) by using a $10 \mathrm{~cm}$ visual 
TABLE 1 Clinical features of fatal asthma children included in the study

\begin{tabular}{|c|c|c|c|c|c|c|c|}
\hline Sex & $\begin{array}{l}\text { Age at onset } \\
\text { of symptoms } \\
\text { (y) }\end{array}$ & $\begin{array}{l}\text { Age at } \\
\text { diagnosis } \\
\text { (y) }\end{array}$ & $\begin{array}{l}\text { Age of } \\
\text { death } \\
\text { (y) }\end{array}$ & $\begin{array}{l}\text { Treatment } \\
\text { delay }(y)\end{array}$ & ICS & $\begin{array}{l}\text { Other } \\
\text { asthma } \\
\text { therapy }\end{array}$ & $\begin{array}{l}{ }^{*} \text { Tissue } \\
\text { eosinophilia } \\
\text { (cells } / \mathrm{mm}^{2} \text { ) }\end{array}$ \\
\hline $\mathrm{F}$ & 2.2 & - & 6 & 3.8 & No & No & 20 \\
\hline $\mathrm{F}$ & 1.1 & - & 1.9 & 0.8 & No & No & 27 \\
\hline$M$ & 0.5 & 3 & 18.9 & 6.5 & No & $\mathrm{T}, \mathrm{C}$ & 65 \\
\hline$M$ & - & - & 0.9 & - & - & - & 1240 \\
\hline$M$ & 1.1 & 1.5 & 1.9 & 0.4 & No & No & 77 \\
\hline $\mathrm{F}$ & 0.7 & 0.8 & 2.6 & 0.1 & Yes & $\mathrm{T}, \mathrm{C}$ & 240 \\
\hline$M$ & 1 & 1.3 & 18.1 & 12 & No & $\mathrm{T}$ & 653 \\
\hline$M$ & 1 & 3 & 19.5 & 3.0 & Yes & $\mathrm{T}, \mathrm{C}$ & 360 \\
\hline$M$ & 0.7 & 0.9 & 2.4 & 0.2 & Yes & $T$ & 320 \\
\hline $\mathrm{F}$ & 1.5 & 1.7 & 1.8 & 0.2 & No & $T$ & 700 \\
\hline$M$ & 15 & 17.8 & 18.7 & 2.8 & Yes & LABA & 1 \\
\hline$M$ & 0.8 & 0.9 & 3.6 & 0.1 & Yes & C & 467 \\
\hline
\end{tabular}

*Data from reference ${ }^{12}$. (F: female; M:male; ICS: inhaled corticosteroids; T: teophyllin; C: cromoglycate; LABA: long-acting beta-agonists) analogue scale ranging from "not bothered at all" to "extremely bothered." Prior to the nasal challenge (day 0), a mucosal biopsy was obtained under topical anaesthesia (tetracaine/adrenaline) from the lower turbinate of one nostril. The allergen challenge was thereafter performed on the other nasal cavity. The second biopsy was obtained under topical anaesthesia 24 hours after the last allergen dose from the lower turbinate of the challenged nostril. No biopsy-related complications occurred.

\section{5 | Tissue preparation for immunohistology}

Multi-colour immunofluorescence was performed as described elsewhere. ${ }^{16}$ The monoclonal antibodies (mAbs) used are listed in Tables S1 and S2. Concentration-matched irrelevant isotype antibodies were used as negative controls. For immunohistochemical localization of S100A8/A9, a DAKO EnVision peroxidase/DAB kit (Agilent Kista, Sweden) was applied.

\section{6 | Immunofluorescence microscopy}

The slides were examined by IEG at $\times 60$ magnification with a confocal microscope. To determine cell densities in the lamina propia of nasal, bronchial and bronchiolar mucosa and in the alveolar compartment, a minimum of 6 representative fields $\left(0.045 \mathrm{~mm}^{2}\right.$ each) with preserved tissue architecture were acquired. These fields did not include portions of respiratory epithelium. To calculate cell densities, all the cells within the acquired fields displaying a specific phenotype were enumerated. The positivity threshold for every marker was determined by the signal emitted by the corresponding isotype control antibody (Figure S2). For illustration purposes, images were captured using the same confocal microscope (Olympus FV1000; Olympus, Hamburg, Germany).

\subsection{Flow cytometric analysis of blood monocytes}

Peripheral blood mononuclear cells (PBMCs) were obtained by density centrifugation (Lymphoprep, StemCell Technologies) from three healthy blood donors. PBMCs were then stained for surface and intracellular markers (antibodies listed in Table S3) using BD Cytofix/ Cytoperm kit and subsequently analysed by flow cytometry (LSR Fortessa, BD Biosciences, Franklin Lakes NJ). Irrelevant isotype- and concentration-matched antibodies were used as controls.

\subsection{Statistics}

GraphPad Prism version 5.0 (GraphPad Software, La Jolla, CA) was used for statistical analysis. The distribution of the samples was assessed using the Kolmogorov-Smirnov test. Comparisons between normally distributed samples were made by Student's $t$ test for either paired or unpaired samples. For samples not normally distributed, either the Kruskal-Wallis or Wilcoxon test was used. A Pvalue of $<0.05$ was considered significant.

\section{RESULTS}

\subsection{S100A8/A9 identifies newly recruited monocytes in allergic airway inflammation}

Monocytes are phenotypically very similar to tissue-resident macrophages, and there are currently no established protocols to distinguish monocytes from macrophages in FFPE sections of the 
respiratory mucosa. When studying the turnover of alveolar macrophages in transplanted lungs using FFPE specimens, we recently found that incoming recipient monocytes selectively expressed the antimicrobial protein S100A8/A9, whereas resident alveolar macrophages from the donor were uniformly S100A8/A9-negative. ${ }^{17}$ Furthermore, we recently reported that blood monocytes constitutively migrate into the intestinal mucosa where they develop into mature macrophages, with a progressive loss of S100A8/A9 expression as part of the differentiation process. ${ }^{18}$ To test the applicability of this marker in an airway allergy setting, we examined FFPE specimens of the nasal mucosa obtained from an experimental in vivo model of AR (Figure S1) in which all allergen-challenged patients experienced typical allergic symptoms and increase in the density of mucosal eosinophils (Figure S3). We have recently shown that monocytes rapidly accumulate in experimentally induced $A R$ using cryo-preserved tissue material, ${ }^{10}$ but in the latter study, we had no positive marker to identify monocytes and used DC-SIGN/CD209 to distinguish monocytes (negative) from macrophages (positive). Co-staining for S100A8/A9 and the pan monocyte/macrophage marker CD68, together with assessments of nuclear morphology to exclude granulocytes, on FFPE sections revealed few double-positive cells at baseline (Figure $1 \mathrm{~A}-\mathrm{C}$ ), but following two days of allergen provocation, there was a striking 11-fold increase in CD68+S100A8/A9+ monocytes, whereas the number of CD68+S100A8/A9- macrophages was unchanged (Figure 1A-C). Of note, there was a correlation between the accumulating monocytes and eosinophils in the nasal mucosa of AR patients $(r=0.5 ; P=0.001)$, suggesting that monocyte recruitment associates with markers of airway allergic inflammation. This observation was consistent with our previous study, ${ }^{10}$ and further substantiated the finding that monocytes are recruited in large numbers to the challenged nasal mucosa of AR patients, whereas the density of macrophages remains unchanged. The observation furthermore demonstrates the applicability of using S100A8/A9 as a marker to identify newly recruited monocytes in FFPE tissues.

\subsection{Monocytes accumulate in the lower airways of children and adolescents with fatal asthma attacks}

To examine whether monocytes are recruited to the inflamed lungs of asthmatics, FFPE lung biopsies from children and adolescent with fatal asthma and lung specimens from age-matched control individuals were analysed. Co-staining for CD68 and S100A8/A9 revealed a 5 -fold higher density $(P=0.0002)$ of monocytes in the lamina propria of the bronchi and bronchioles in asthmatic subjects compared to controls (Figure 2A). The number of CD68+S100A8/A9- macrophages was also higher in asthmatics, yet the difference was less pronounced (3-fold increase, $P=0.0028$ ) (Figure 2B). Importantly, the number of accumulating monocytes and macrophages was not
(A)

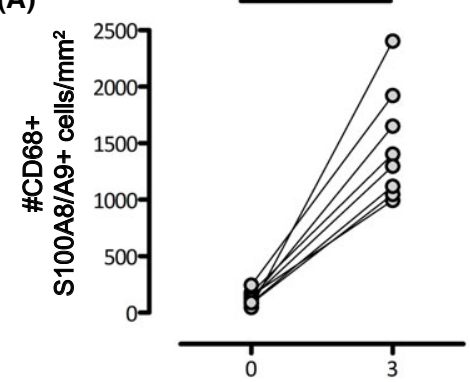

Days on allergen challenge

(C)

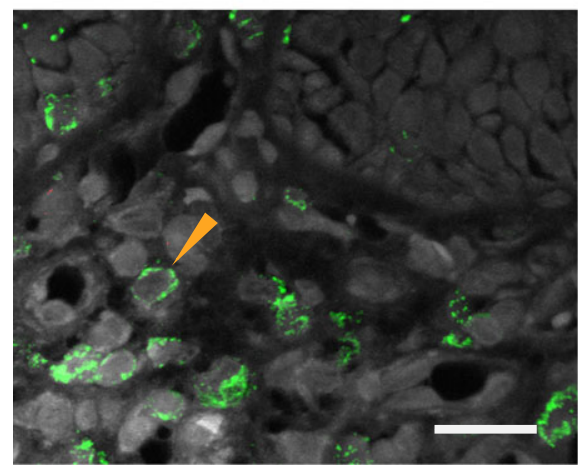

Baseline
(B)

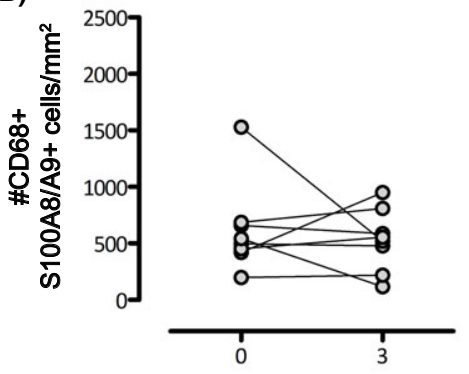

S100A8/A9 CD68

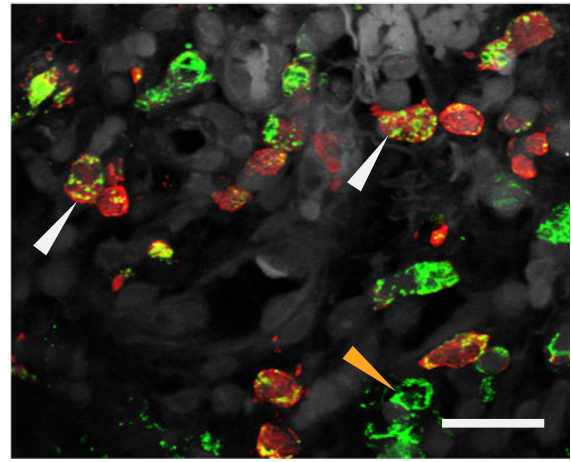

Post-challenge
FIGURE 1 Co-staining for CD68 and S100A8/A9 discriminates recruited monocytes from resident macrophages in the airways of allergic patients. Density of CD68+S100A8/A9+monocytes (A) and CD68+S100A8/A9-macrophages (B) in the nasal mucosa of allergic rhinitis patients at baseline and after 2 days of allergen challenge. C, Two-colour immunofluorescence staining of CD68 and S100A8/A9 at baseline and after provocation. The yellow and white arrowheads indicate macrophages and monocytes, respectively ( $* * * P<0.001)$. Scale bars: $30 \mu \mathrm{m}$ 

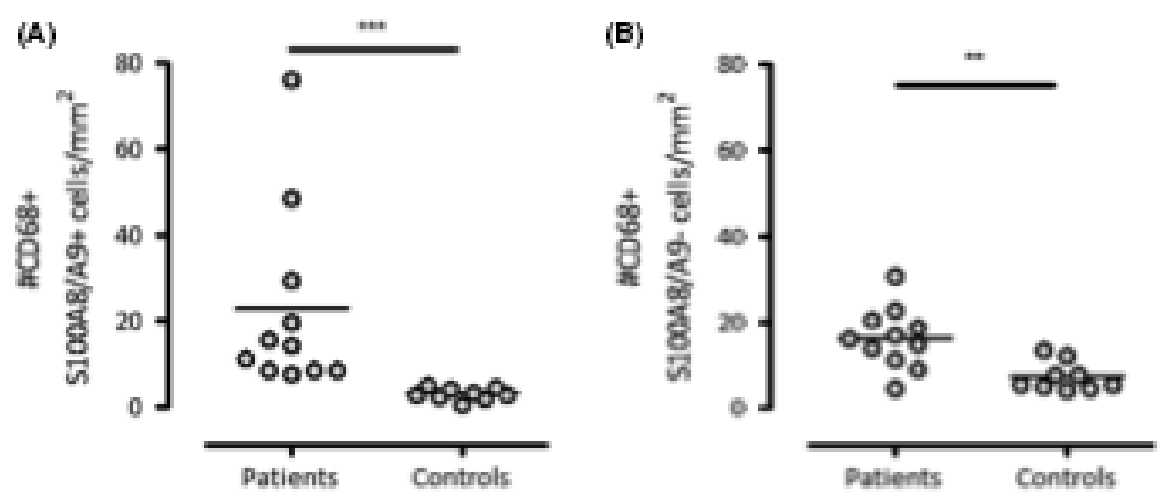

(C)

FIGURE 2 Both monocytes and macrophages are increased in the bronchial mucosa of children with fatal asthma attacks. Density of CD68+S100A8/ $\mathrm{A} 9+$ monocytes $(\mathrm{A})$ and $\mathrm{CD} 68+\mathrm{S} 100 \mathrm{~A} 8 / 9$ macrophages $(B)$ in the lamina propia of bronchial mucosal tissue from asthmatic children and non-atopic controls. Twocolour immunofluorescence staining of CD68 and S100A8/A9 on FFPE section from control and asthmatic patient (C). Yellow and white arrowheads indicate macrophages and monocytes. S100A8/ A9+CD68-cells represent granulocytes $(* * P<0.01 * * * P<0.001)$. Scale bars: $30 \mu \mathrm{m}$

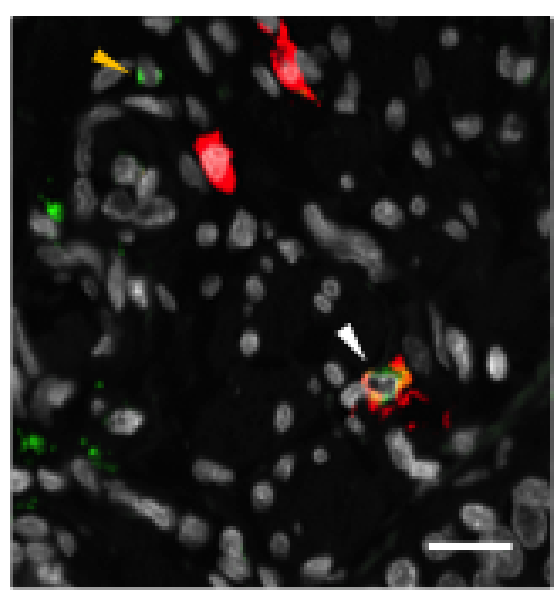

Control

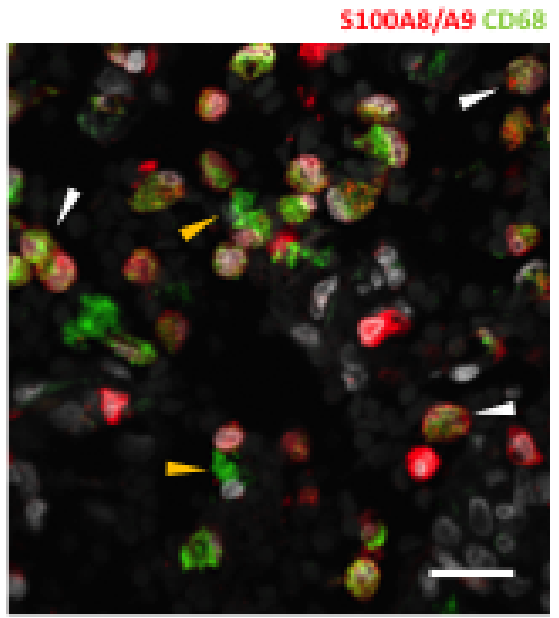

Asthmatic patient
(A)

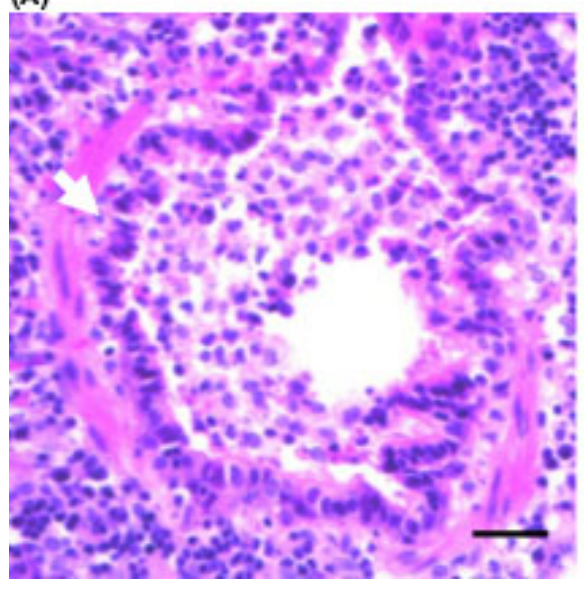

(B)

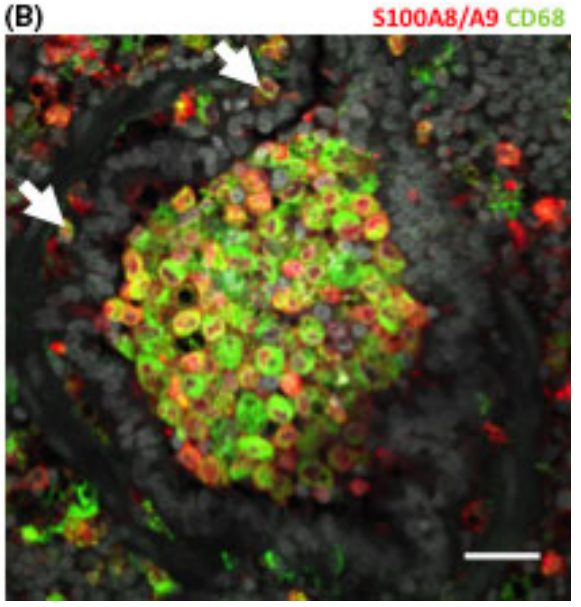

FIGURE 3 Aggregates of monocytes obstruct the airways of children with fatal asthma attacks. Haematoxylin-eosin staining of a FFPE section from a child suffering from fatal asthma attack showing a bronchiole with abundant mononuclear cells in the airway lumen (A).Twocolour immunofluorescence staining of CD68 and S100A8/A9 in an adjacent section (B) to that shown in A. The white arrows mark intraepithelial S100A8/A9+CD68+ mononuclear cells (monocytes). S100A8/A9+CD68-cells represent granulocytes. Scale bars: $50 \mu \mathrm{m}$

influenced by disease duration, age at death or the treatment with inhaled corticosteroids (data not shown). A recent study examining the same tissue samples demonstrated increased thickness of the basement membrane, and higher numbers of eosinophils, $\mathrm{T}$ lymphocytes and smooth muscle cells in asthmatics as compared with controls ${ }^{12}$ (Table 1). There was no association between elicited monocytes and $\mathrm{T}$ lymphocytes or smooth muscle cells in the bronchial mucosa of asthmatics (data not shown), but there was a 
(A)
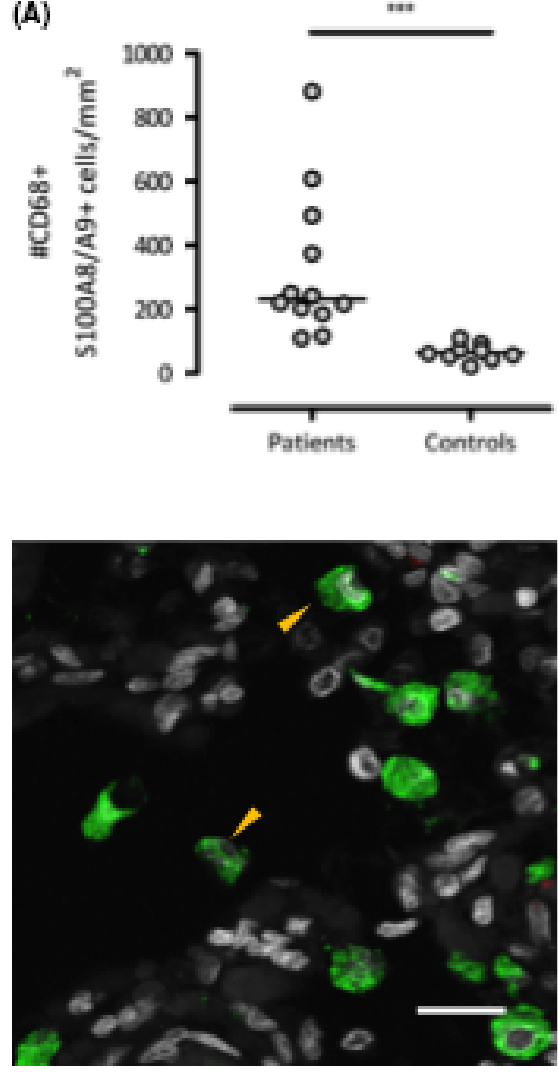

Control
(B)
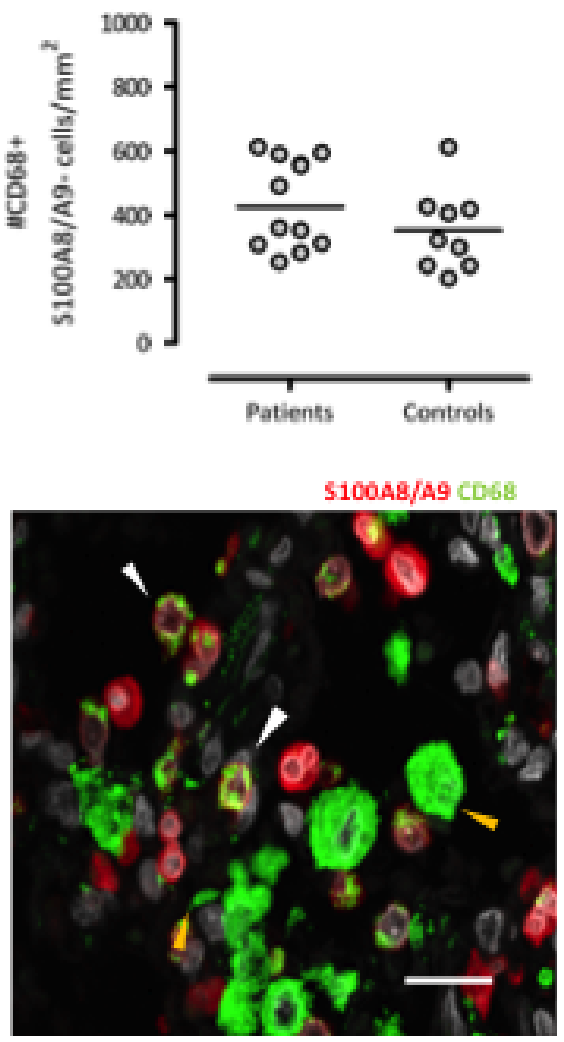

Asthmatic patient
FIGURE 4 Monocytes, but not alveolar macrophages, are increased in the lung parenchyma of children with fatal asthma attacks. Density of CD68+S100A8/A9+ monocytes (A) and CD68+S100A8/A9macrophages (B) in the parenchyma of asthmatic children and non-atopic controls. Two-colour immunofluorescence staining of CD68 and S100A8/A9 on FFPE section from control and asthmatic patient samples. Yellow and white arrowheads indicate macrophages and monocytes, respectively. S100A8/A9+CD68- cells are granulocytes $(* * * P<0.001)$. Scale bars: $50 \mu \mathrm{m}$ significant correlation between monocytes and eosinophils $(r=0.684 ; P=0.001)$ and between monocytes and the thickness of the basement membrane $(r=0.639 ; P=0.002)$.

Interestingly, in 8 of 12 asthma subjects, but not in the controls, cellular aggregates containing CD68+S100A8/A9+ monocytes obstructing the lumen of bronchioles were found (Figure 3). Moreover, single monocytes between the basement membrane and the epithelial layer were observed (Figure 3B). Also in the parenchyma of asthmatic individuals, the density of CD68+S100A8/A9+ monocytes was higher (4-fold, $P=0.0002$ ) than in control subjects (Figure $4 A$ ), but the number of CD68+S100A8/A9- alveolar macrophages was comparable between the two groups (Figure 4B). Most parenchymal monocytes in the lung of asthmatics appeared to reside within the interstitium (Figure S5), but monocytes were also observed within the alveolar lumen (Figure 4B). As in the bronchi, neither the disease duration, age at death nor the anti-asthma therapy affected the accumulation of monocytes in the parenchyma of asthmatics (data not shown). Together, these observations imply that recruited monocytes are able to migrate from the vessels in the lamina propia to the airway lumen.

\section{3 | Monocytes express MMP10 in allergic airway inflammation}

We recently performed global transcriptomic analysis of sorted CD45+HLA-DR+ APCs in a short-term (48 hours) experimental model of human $A R$, in which we found a strong upregulation of several genes including matrix metalloproteinase 10 (MMP10) after provocation (Eguiluz-Gracia et al, unpublished). MMP10, also termed stromelysin-2, is an endopeptidase involved in the breakdown of extracellular matrices (ECMs) by cleaving proteoglycans and fibronectin. ${ }^{19}$ MMP10 is also utilized by mouse bone marrow-derived macrophages to migrate through ECMs. $^{20}$ Interestingly, sorted peripheral blood monocytes from healthy adults uniformly expressed MMP10 (Figure 5A), and we thus hypothesized that accumulating monocytes may use this protein to migrate within the inflamed tissue. To this end, we performed three-colour immunofluorescence in situ staining for CD68, S100A8/A9 and MMP10. Of note, we found that many CD68+S100A8/A9+ monocytes both in the lumen of small vessels and in the lamina propia expressed MMP10 in allergen-challenged AR patients (Figure 5B). As previously reported, monocytes are quite scarce in nasal mucosal samples obtained before provocation, ${ }^{10}$ and only occasional MMP10+ monocytes were seen at baseline (Figure S4). We then examined the occurrence of MMP10+ monocytes in FFPE tissue from asthmatic patients. In the lamina propia of bronchi and bronchioles, a median of $70 \%$ of CD68+S100A8/A9+ monocytes expressed MMP10, and the density of MMP10+ monocytes was 7-fold higher $(P=0.0002)$ than in control individuals (Figure $5 C-E)$. Also in the lung parenchyma, 75\% of CD68+S100A8/A9+ monocytes expressed MMP10, and the density was 4-fold higher $(P=0.0001)$ than in controls (Figure 5F,H). Taken together, these findings suggest that MMP10 expression might contribute to monocyte migration in the respiratory tract. 
(A)

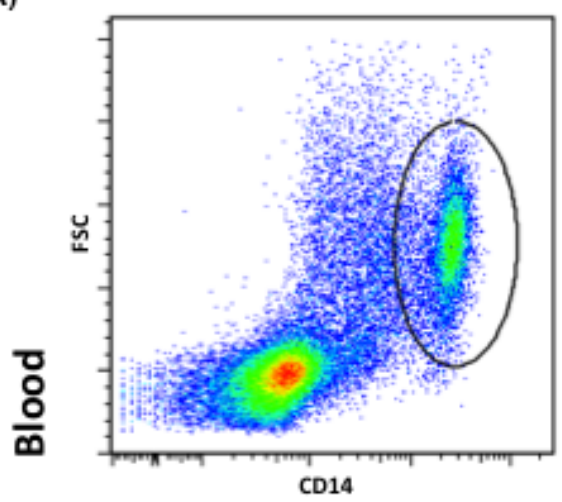

(B)

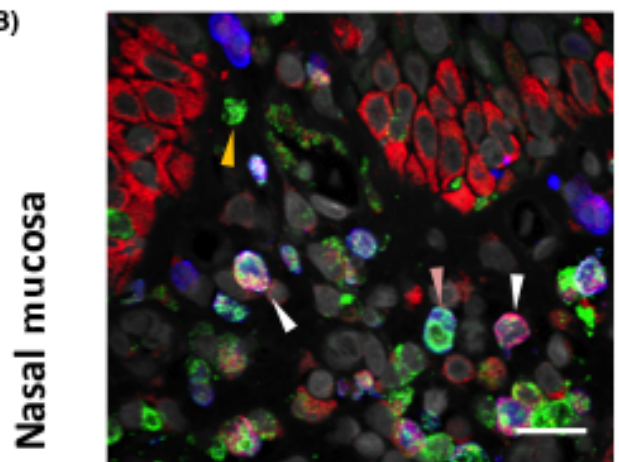

Lamina propia

(C)
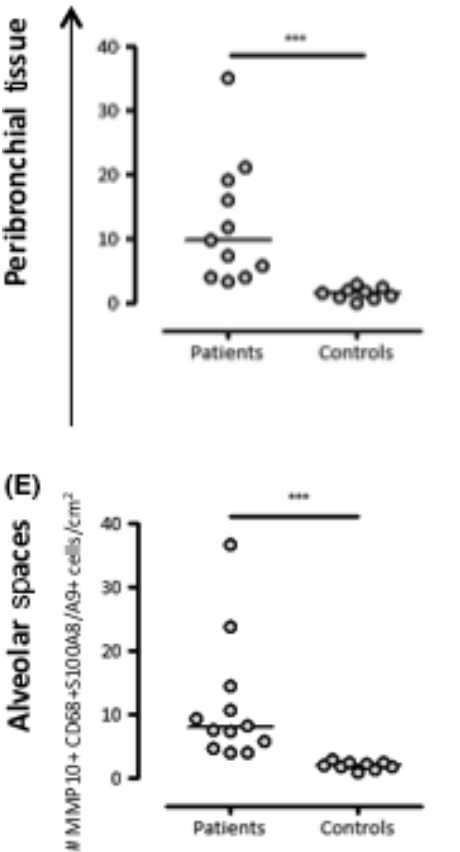

(D)

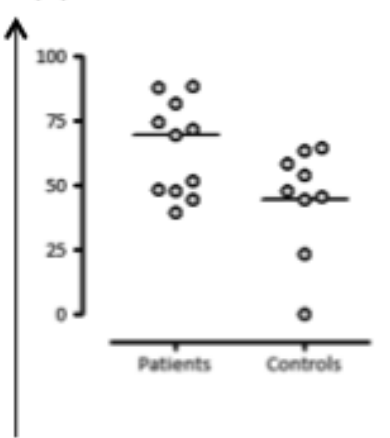

(F)

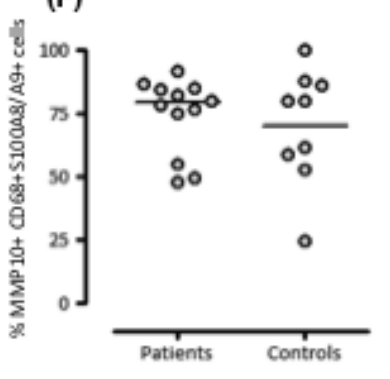

Isotype control MMP10

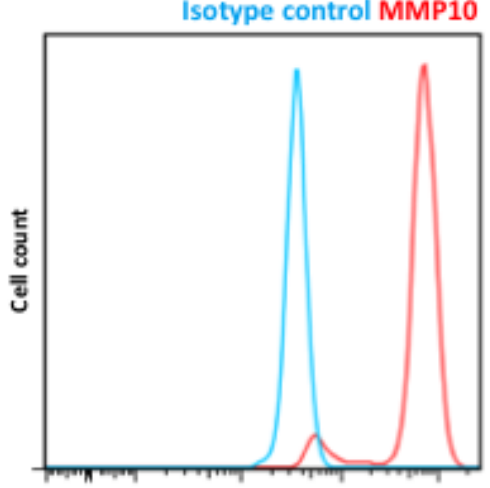

MMP-10 S100A8/A9 CD 68

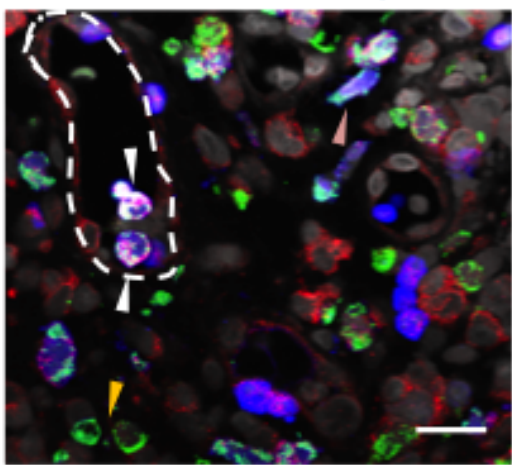

Intravascular monocytes

(G)
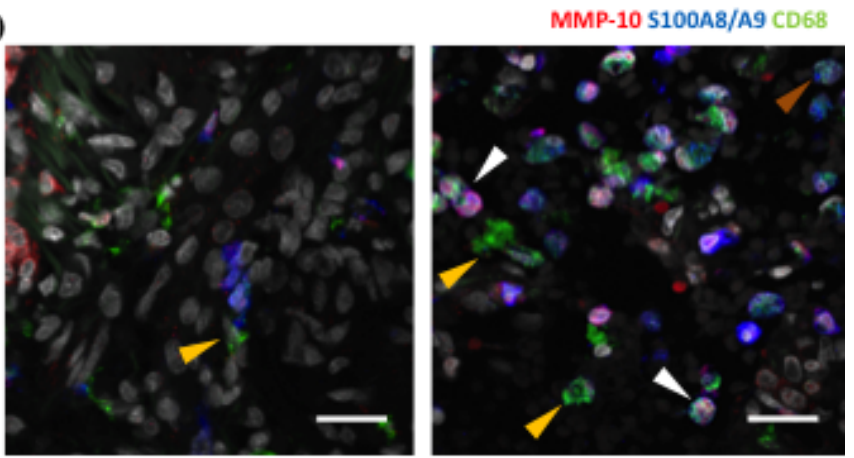

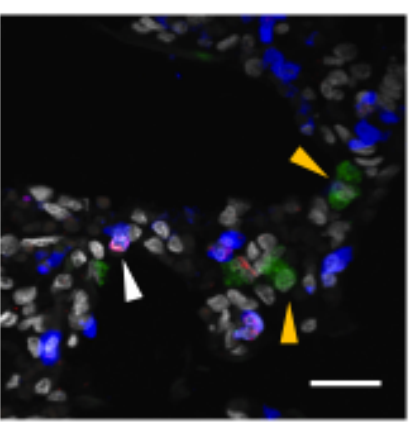

Control subjects

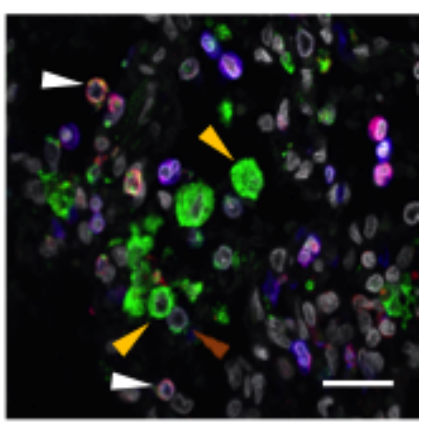

Asthmatic patients

FIGURE 5 The majority of monocytes in the respiratory tract of asthmatics express matrix metalloproteinase 10 (MMP10). PBMCs from a healthy donor were gated for CD14 (left panel), followed by intracellular staining of MMP10 vs isotype antibody (right panel) (A). Three-colour immunofluorescence staining of CD68, S100A8/A9 and MMP10 on FFPE section of the nasal mucosa from an AR patient after 2 days of allergen challenge (B). Density (C, E) and proportion (D, F) of MMP10+ monocytes in the bronchial lamina propia (C, D) and alveolar tissue (E, F) of asthma patients and non-atopic controls. Three-colour immunofluorescence staining of CD68, S100A8/A9 and MMP10 on FFPE sections in the bronchial lamina propia and pulmonary alveoli of representative asthmatic and control samples (G). The yellow, white and brown arrowheads indicate alveolar macrophages, MMP10+ monocytes and MMP10- monocytes, respectively. S100A8/A9+CD68-cells are granulocytes (***P<0001). Scale bars: $30 \mu \mathrm{m}$ [Colour figure can be viewed at wileyonlinelibrary.com] 


\section{DISCUSSION}

In an experimental model of human $\mathrm{AR}$, we have previously reported that $\mathrm{CD} 1 \mathrm{c}+\mathrm{CDCs}$ and plasmacytoid (p)DCs accumulated in the nasal mucosa after long-term allergen challenge. However, tissue DCs and macrophages were both strongly outnumbered by a robust and rapid influx of proinflammatory monocytes. ${ }^{10,15,21}$ Recruitment of CD1c+ $\mathrm{cDCs}$ and $\mathrm{pDCs}$ has also been found in the allergic inflammation of asthmatics, ${ }^{5,6,22}$ but whether monocytes contribute to the inflammatory reaction in allergic asthma has not been examined. In this study, we demonstrate that tissue recruitment of monocytes is a prominent part of the inflammatory reaction in the lungs of children and adolescents with fatal asthma attacks. The majority of the accumulating monocytes expressed MMP10, a molecule potentially involved in tissue migration.

The robust recruitment of monocytes to the lungs of paediatric and adolescent patients with fatal asthma is consistent with a previous study of children hospitalized due to asthma exacerbations, which displayed increased numbers of activated monocytes in the circulation. ${ }^{9}$ Furthermore, in a mouse model of asthma, incoming monocytes were identified as crucial local orchestrators during the effector phase of allergic inflammation, ${ }^{8}$ whereas cDCs were more important for the initial sensitization process in the draining lymph nodes. Thus, it is reasonable to suggest that the massively elicited monocytes observed in the patients may play a similar role. Our study did not reveal striking differences in the number of lung-resident macrophages between asthmatics and non-atopic controls, suggesting that these cells do not have a prominent role during fatal asthma attacks. Although our study is limited by a relatively small sample size of autopsy material, the expression levels of the markers used to identify cell subsets were reportedly not significantly altered within the 48 hours' post-mortem. ${ }^{23,24}$

In this study, it was not possible to identify the final fate of the recruited monocytes. Mouse models indicate that most monocytes recruited during acute lung injury experience apoptosis as inflammation resolves. ${ }^{25}$ However, a recent study of human duodenal transplants suggested that monocytes migrating to the mucosae during homoeostasis differentiate into macrophages, characterized by a maturation process involving S100A8/A9 downregulation and the progressive decrease in responsiveness to pro-inflammatory stimuli. $^{18}$ Moreover, inflammation of mucosal tissues supports a highly increased recruitment of monocytes and alters their final differentiation from regulatory macrophages to inflammatory monocytes, ${ }^{8,26,27}$ underscoring that the developmental fate of incoming monocytes is highly dependent on the tissue environment upon migration. Mouse models also indicate that lung-resident DCs might partially arise from blood monocytes during the steady state, ${ }^{28,29}$ but robust markers applicable to histological sections for separation of monocytederived macrophages and DCs are not available. Moreover, lymph node homing capacity and T-cell stimulatory potential cannot be inferred from tissue staining. Although functional data are lacking, our finding of monocytes accumulation in the lower airways of fatal asthma attacks more likely reflects that inflammatory events in the asthmatic lung promote a large influx of monocytes that become "arrested" in a pro-inflammatory state. Importantly, the correlation between monocyte infiltration in the bronchial mucosa and the thickness of the basement membrane of the bronchial epithelium suggests that these cells might contribute not only to the inflammatory reaction, but also to the remodelling processes.

We also found that CD68+S100A8/A9+ monocytes accumulated in the alveolar walls of asthmatics, and that some of them were able to migrate to the airway lumen. Previous studies have reported an influx of lymphocytes and eosinophils into alveolar tissue of asthma patients. ${ }^{30}$ The recruitment of monocytes to the lung parenchyma after allergen challenge had been previously identified in mouse models of asthma, ${ }^{31}$ but data from human asthmatic lungs have been lacking. Together, these findings identify the alveolar compartment as an active site of inflammation during severe asthma. A recent study showed that adult patients with fatal asthma attacks display increased numbers of myofibroblasts in the lung parenchyma, ${ }^{32}$ suggesting that this anatomical compartment could also be affected by the tissue remodelling process.

Migration of cells within the tissue depends on several factors including the expression of MMPs with the ability to cleave the large biomolecules of the ECM, thus facilitating the movement of migrating cells. $^{20,33}$ Interestingly, we found that most of the accumulating monocytes expressed MMP10. Recently, MMP10 was reported to be expressed by mouse bone marrow-derived macrophages during experimental models of inflammation ${ }^{20,34}$; thus, MMP10 might play a role in the motility of monocytes that accumulated in the peripheral airways.

Our results suggest that CD68+S100A8/A9+ monocytes constitute potential therapeutic targets for airway allergy. In this regard, the inhibition of monocytes is probably one of the multiple actions of inhaled corticosteroids in asthma. ${ }^{35}$ Moreover, mouse models suggest that blocking monocyte recruitment by targeting CCR2 reduces lung inflammation in asthma. ${ }^{8}$ Additionally, MMP10 antagonism might substantially reduce monocyte migration within the inflamed lungs. Further research exploring the therapeutic potential of antagonizing the recruitment and migration of monocytes in human airway allergy may provide new therapeutic approaches for severe asthma.

\section{ACKNOWLEDGEMENTS}

The authors thank Inge Dahle for providing us with the S100A8/A9 antibody, and Kjersti Thorvaldsen Hagen for her excellent technical assistance.

\section{CONFLICT OF INTEREST}

The authors declare no conflict of interest.

\section{ORCID}

Espen S. Baekkevold (iD http://orcid.org/0000-0002-5890-9108

\section{REFERENCES}

1. Szefler SJ, Chmiel JF, Fitzpatrick AM, et al. Asthma across the ages: knowledge gaps in childhood asthma. J Allergy Clin Immunol. 2014;133:3-13; quiz 14. 
2. Fu LS, Tsai MC. Asthma exacerbation in children: a practical review. Pediatr Neonatol. 2014;55:83-91.

3. Chow A, Brown BD, Merad M. Studying the mononuclear phagocyte system in the molecular age. Nat Rev Immunol. 2011;11:788-798.

4. Lambrecht $\mathrm{BN}$, Hammad $\mathrm{H}$. Lung dendritic cells in respiratory viral infection and asthma: from protection to immunopathology. Annu Rev Immunol. 2012;30:243-270.

5. Jahnsen FL, Moloney ED, Hogan T, Upham JW, Burke CM, Holt PG. Rapid dendritic cell recruitment to the bronchial mucosa of patients with atopic asthma in response to local allergen challenge. Thorax. 2001;56:823-826.

6. Upham JW, Denburg JA, O'Byrne PM. Rapid response of circulating myeloid dendritic cells to inhaled allergen in asthmatic subjects. Clin Exp Allergy. 2002;32:818-823.

7. Tashiro H, Takahashi K, Hayashi S, et al. Interleukin-33 from monocytes recruited to the lung contributes to house dust mite-induced airway inflammation in a mouse model. PLOS ONE. 2016;11: e0157571.

8. Plantinga $M$, Guilliams $M$, Vanheerswynghels $M$, et al. Conventional and monocyte-derived $\mathrm{CD} 11 \mathrm{~b}(+)$ dendritic cells initiate and maintain $\mathrm{T}$ helper 2 cell-mediated immunity to house dust mite allergen. Immunity. 2013;38:322-335.

9. Subrata LS, Bizzintino J, Mamessier E, et al. Interactions between innate antiviral and atopic immunoinflammatory pathways precipitate and sustain asthma exacerbations in children. J Immunol 2009;183:2793-2800.

10. Eguiluz-Gracia I, Bosco A, Dollner R, et al. Rapid recruitment of CD14 monocytes in experimentally induced allergic rhinitis in human subjects. J Allergy Clin Immunol. 2016;137:1872-1881.

11. Malmstrom K, Kaila M, Korhonen K, et al. Mechanical ventilation in children with severe asthma. Pediatr Pulmonol. 2001;31:405-411.

12. Malmstrom K, Lohi J, Sajantila A, et al. Immunohistology and remodeling in fatal pediatric and adolescent asthma. Respir Res. 2017;18:94

13. Keglowich LF, Borger P. The Three A's in Asthma - Airway Smooth Muscle, Airway Remodeling \& Angiogenesis. Open Respir Med J. 2015;9:70-80.

14. Bousquet J, Van Cauwenberge $P$, Khaltaev N. Allergic rhinitis and its impact on asthma. J Allergy Clin Immunol. 2001;108:S147-S334.

15. Jahnsen FL, Lund-Johansen F, Dunne JF, Farkas L, Haye R, Brandtzaeg P. Experimentally induced recruitment of plasmacytoid (CD123high) dendritic cells in human nasal allergy. J Immunol 2000;165:4062-4068.

16. Jahnsen FL, Gran E, Haye R, Brandtzaeg P. Human nasal mucosa contains antigen-presenting cells of strikingly different functional phenotypes. Am J Respir Cell Mol Biol. 2004;30:31-37.

17. Eguiluz-Gracia I, Schultz HH, Sikkeland LI, et al. Long-term persistence of human donor alveolar macrophages in lung transplant recipients. Thorax. 2016;71:1006-1011.

18. Bujko A, Atlasy N, Landsverk OJB, et al. Transcriptional and functional profiling defines human small intestinal macrophage subsets. $J$ Exp Med. 2018;215:441-458.

19. Verma RP, Hansch C. Matrix metalloproteinases (MMPs): chemicalbiological functions and (Q)SARs. Bioorg Med Chem. 2007;15:22232268.

20. Murray MY, Birkland TP, Howe JD, et al. Macrophage migration and invasion is regulated by MMP10 expression. PLOS ONE. 2013;8: e63555.

21. Melum GR, Farkas L, Scheel C, et al. A thymic stromal lymphopoietin-responsive dendritic cell subset mediates allergic responses in the upper airway mucosa. J Allergy Clin Immunol. 2014;134(613-21): e7.
22. Bratke $K$, Lommatzsch $M$, Julius $P$, et al. Dendritic cell subsets in human bronchoalveolar lavage fluid after segmental allergen challenge. Thorax. 2007;62:168-175.

23. Holbrook EH, Wu E, Curry WT, Lin DT, Schwob JE. Immunohistochemical characterization of human olfactory tissue. Laryngoscope. 2011;121:1687-1701.

24. Guarner J, Paddock CD, Shieh WJ, et al. Histopathologic and immunohistochemical features of fatal influenza virus infection in children during the 2003-2004 season. Clin Infect Dis. 2006;43:132140.

25. Janssen WJ, Barthel L, Muldrow A, et al. Fas determines differential fates of resident and recruited macrophages during resolution of acute lung injury. Am J Respir Crit Care Med. 2011;184:547-560.

26. Rivollier A, He J, Kole A, Valatas V, Kelsall BL. Inflammation switches the differentiation program of Ly6Chi monocytes from antiinflammatory macrophages to inflammatory dendritic cells in the colon. J Exp Med. 2012;209:139-155.

27. Bain $\mathrm{CC}$, Scott $\mathrm{CL}$, Uronen-Hansson $\mathrm{H}$, et al. Resident and proinflammatory macrophages in the colon represent alternative context-dependent fates of the same Ly6Chi monocyte precursors. Mucosal Immunol. 2013;6:498-510.

28. Schraml BU, van Blijswijk J, Zelenay S, et al. Genetic tracing via DNGR-1 expression history defines dendritic cells as a hematopoietic lineage. Cell. 2013;154:843-858.

29. Kopf M, Schneider C, Nobs SP. The development and function of lung-resident macrophages and dendritic cells. Nat Immunol. 2015;16:36-44.

30. Kraft M, Martin RJ, Wilson S, Djukanovic R, Holgate ST. Lymphocyte and eosinophil influx into alveolar tissue in nocturnal asthma. Am J Respir Crit Care Med. 1999;159:228-234.

31. Veres TZ, Voedisch S, Spies E, Valtonen J, Prenzler F, Braun A. Aeroallergen challenge promotes dendritic cell proliferation in the airways. J Immunol 2013;190:897-903.

32. Boser SR, Mauad T, Araujo-Paulino BB, et al. Myofibroblasts are increased in the lung parenchyma in asthma. PLOS ONE. 2017;12: e0182378.

33. Lorenc VE, Jaldin-Fincati JR, Luna JD, Chiabrando GA, Sanchez MC. IGF-1 regulates the extracellular level of active MMP-2 and promotes muller glial cell motility. Invest Ophthalmol Vis Sci. 2015;56:6948-6960.

34. Rohani MG, McMahan RS, Razumova MV, et al. MMP-10 regulates collagenolytic activity of alternatively activated resident macrophages. J Invest Dermatol. 2015;135:2377-2384.

35. Heffler E, Madeira LNG, Ferrando M, et al. Inhaled Corticosteroids Safety and Adverse Effects in Patients with Asthma. J Allergy Clin Immunol Pract. 2018;6:776-781.

\section{SUPPORTING INFORMATION}

Additional supporting information may be found online in the Supporting Information section at the end of the article.

How to cite this article: Eguíluz-Gracia I, Malmstrom K, Dheyauldeen SA, et al. Monocytes accumulate in the airways of children with fatal asthma. Clin Exp Allergy. 2018;48:16311639. https://doi.org/10.1111/cea.13265 